\section{Satisfação dos usuários assistidos nos Centros de Especialidades Odontológicas do Município do Recife, Pernambuco, Brasil}

\author{
Patient satisfaction at Specialized Dental Clinics \\ in Recife, Pernambuco State, Brazil
}

\begin{abstract}
This study evaluated patient satisfaction at four specialized dental clinics in Recife, Pernambuco State, Brazil. Data were collected through a standardized self-applied individual questionnaire with 272 patients, to evaluate user satisfaction with public dental services. The findings indicated a positive evaluation on most dimensions, except for accessibility. Significant differences were observed between the services on the following dimensions: patient-dentist relationship ( $p=0.001)$; patient-dental assistant relationship ( $p=0.007) ;$ effectiveness ( $p=0.014$ ) accessibility/ waiting time ( $p=0.018)$; physical environment/ reception room cleanness ( $p=0.004)$; acceptability ( $p=0.029)$; and effectiveness/tooth appearance ( $p=0.029)$, effectiveness/chewing capacity $(p<0.001)$. In conclusion, most users were satisfied with the dental care, and satisfaction differed between services.
\end{abstract}

Patient Satisfaction; Quality of Health Care; Oral Health
Ana Carolina Silva de Lima 1

Etenildo Dantas Cabral 2

Marcia Maria Vendiciano Barbosa Vasconcelos 1

Os Centros de Especialidades Odontológicas são estabelecimentos de saúde da atenção secundária, registrados no Cadastro Nacional de Estabelecimento de Saúde (CNES). Devem oferecer, no mínimo, as especialidades de diagnóstico bucal, periodontia, endodontia, cirurgia oral menor e atendimento aos portadores de necessidades especiais. Segundo o critério físico-estrutural, os centros podem ser classificados em tipo I (três cadeiras odontológicas), tipo II (quatro a seis cadeiras odontológicas) e tipo III (no mínimo sete cadeiras) 1 . Atualmente, as Portaria $n^{o}$. 599/GM 1 e Portaria $n^{\circ}$. 600/GM 2 representam o arcabouço jurídico que normatizam a implantação e o financiamento dessas unidades de saúde, respectivamente.

Como serviços de saúde que representam, os Centros de Especialidades Odontológicas devem ser avaliados objetivando a melhoria contínua da qualidade do serviço. Nesse contexto, surgiu a necessidade de avaliar a satisfação do usuário como um indicador de resultado, o qual, oriundo dos conceitos da indústria, vem ganhando importância crescente na área da saúde, em virtude não apenas da competitividade do setor privado, como também da luta constante pelo exercício da cidadania 3 .

Na área de saúde, após a 2a Guerra Mundial, teve início a realização de estudos visando a avaliar a qualidade de serviços. Inicialmente, a ava- 
liação era realizada a partir de aspectos isolados, como controle de infecção hospitalar, padrões de adequação de instalações e sistematização dos procedimentos executados pela enfermagem. Mas, ainda nesse período, um novo componente, o usuário, passou a integrar o processo de avaliação em saúde, como parte interessada e corresponsável pelas questões pertinentes à qualidade de assistência 4,5 .

Primeiramente, Donabedian 6 atribuiu três dimensões à qualidade: conhecimento técnicocientífico, relações interpessoais entre os profissionais e o paciente e amenidades, isto é, condições de conforto e estética das instalações e equipamentos no local onde a prestação ocorre. Em outro modelo, ampliou o conceito de qualidade, utilizando o que chamou de "sete pilares da qualidade": eficácia, efetividade, eficiência, otimização, aceitabilidade, legitimidade e eqüidade 3,6,7. Sendo assim, considerando o nível de satisfação do usuário, a avaliação da qualidade de assistência à saúde deve aferir o ajuste do serviço à demanda e ser capaz de detectar em que medida esse serviço atende às expectativas do paciente. Esta avaliação é considerada imprescindível para orientar gestores quanto às ações de saúde desenvolvidas 8,9,10.

Então, no que se refere ao valor dos estudos da qualidade em saúde, a satisfação do usuário pode ser considerada tão indispensável para a avaliação da qualidade como o desempenho e a administração dos sistemas de cuidados de saúde. As percepções dos pacientes são igualmente vitais para a avaliação da qualidade 11 .

$\mathrm{Na}$ avaliação da qualidade dos sistemas de saúde de 191 países, realizada pela Organização Mundial da Saúde (OMS), foi observada pouca correlação entre os resultados decorrentes da avaliação técnica e a satisfação dos usuários, uma vez que os melhores sistemas de saúde, de acordo com a OMS, foram avaliados como "ruins" por seus usuários. Contudo, mesmo havendo divergência de percepção, tanto a visão do usuário quanto a dos peritos são importantes, reveladoras e devem ser consideradas em avaliações internacionais dos serviços de saúde 12 .

Em relação à satisfação dos usuários com a assistência à saúde no Brasil, a pesquisa mundial, realizada em 2003, reavivou antigos problemas observados em estudos locais. De acordo com os resultados obtidos, o tempo de espera e a liberdade de escolha do profissional representaram os maiores motivos de insatisfação entre os usuários da rede pública de saúde no país 13 .

Na área da saúde bucal, para a avaliação da qualidade dos atendimentos odontológicos prestados numa universidade do Estado de Santa Catarina, conforme a opinião dos pacientes, os critérios de confiabilidade, presteza, segurança, empatia e tangibilidade foram utilizados como indicadores. No estudo em questão, de forma geral, as manifestações positivas dos usuários sobre o serviço foram superiores às negativas, com ênfase para o bom relacionamento entre o aluno (futuro profissional) e o paciente, na categoria empatia, e o bom atendimento, na categoria confiabilidade. Por outro lado, os tempos de espera para ser chamado para iniciar o tratamento, para aguardar na sala de espera e para finalizar o tratamento representaram as manifestações negativas predominantes 14 .

Apesar de alguns autores 15,16,17 estarem preocupados e já desenvolverem estudos sobre a qualidade em saúde sob a ótica dos usuários, existe uma carência de instrumentos validados para realização das pesquisas, especificamente na área da odontologia, as quais são mais comumente realizadas nas instituições de Ensino Superior 14,18 e em serviços de atenção básica 17,19,20, porém raramente nos serviços de atenção secundária 21.

Buscando a padronização de um instrumento de coleta de dados, foi elaborado e validado no curso de doutorado em odontologia da Universidade de Pernambuco o Questionário de Avaliação da Qualidade dos Serviços de Saúde Bucal (QASSaB) 22, desenvolvido com base no modelo de avaliação da qualidade proposto por Donabedian ${ }^{6}$. Após ter sido submetido a uma análise fatorial, resultou em sete dimensões da qualidade - relações humanas, eficácia, acessibilidade, qualidade técnico-científica, ambiente físico/ limpeza, aceitabilidade e resolutividade.

Diante disso, o objetivo deste estudo foi avaliar a satisfação dos usuários assistidos nos quatro Centros de Especialidades Odontológicas do Município do Recife, Pernambuco, conforme as dimensões da qualidade para avaliação de serviços de saúde bucal do QASSaB, identificando possíveis diferenças entre os resultados obtidos em cada uma dessas unidades de atenção secundária.

\section{Metodologia}

Foi realizado um estudo quantitativo e analítico com um desenho do tipo transversal nos Centros de Especialidades Odontológicas do Recife, capital do Estado de Pernambuco, Região Nordeste do Brasil.

O Município do Recife se encontra habilitado em gestão plena do sistema municipal e apresenta a estratégia de saúde da família como o modelo de reorganização da atenção básica. A cidade possui uma extensão territorial da ordem 
de $217.494 \mathrm{~km}^{2}$, sendo subdividida em 94 bairros, que estão distribuídos em seis Regiões PolíticoAdministrativas (RPA). Na saúde, cada RPA corresponde a um Distrito Sanitário (DS) 23.

Apenas os DS I, III, IV e V possuem Centros de Especialidades Odontológicas que funcionam em instituições tradicionais de atendimento ao usuário. Esses distritos fizeram parte do estudo, respectivamente, por meio dos centros localizados nas seguintes instituições: Policlínica Waldemar de Oliveira, Santo Amaro (CEO A); Centro de Saúde Clementino Fraga, Casa Amarela (CEO B); Policlínica e Centro de Saúde Lessa de Andrade, Madalena (CEO C); Policlínica Agamenon Magalhães, Afogados (CEO D).

Os centros avaliados vêm funcionando como referência de serviço especializado para cada distrito. Eles adotaram uma sistemática similar de funcionamento quanto às especialidades disponibilizadas, turnos de atendimento ao usuário, protocolo de marcação de primeira consulta e cota de atendimento por profissional. Nenhum deles oferece todas as especialidades odontológicas exigidas pelo Ministério da Saúde e, em cada centro, quatro a cinco pacientes são atendidos por especialista.

A população de referência compôs-se de pessoas adultas assistidas nos centros com idade igual ou superior a 18 anos. Para a realização do cálculo do tamanho da amostra, foi utilizado o programa estatístico Epi Info, versão 6.04 (Centers for Disease Control and Prevention, Atlanta, Estados Unidos), utilizando precisão de 5\%, intervalo de confiança de 90\% (IC90\%) e fator de correção 1. Para maximização da amostra, foi considerada uma população infinita e um grau de satisfação médio (prevalência de 50\% para satisfeitos e insatisfeitos com o serviço). Como resultado desse cálculo, o tamanho total da amostra foi de 272 pacientes, sendo 68 respondentes de cada centro. Esse tamanho alcança um poder de teste de $80 \%$ para detectar uma magnitude 0,5 do teste de qui-quadrado com grau de liberdade 6 e um nível de significância de $5 \%$.

A coleta de dados foi realizada por meio de entrevista pessoal padronizada, aplicada pela pesquisadora, dentista e mestranda, numa sala dos próprios centros, resguardando a privacidade do entrevistado. $\mathrm{O}$ formulário da entrevista foi constituído por uma parte semi-estruturada, referindo-se aos dados demográficos e sócio-econômicos do entrevistado, e outra estruturada, o QASSaB 22, sendo alguns termos adaptados para a pesquisa após a realização do projeto-piloto.

O QASSaB 22 foi elaborado e validado no curso de doutorado em odontologia da Universidade de Pernambuco, com base no modelo de avaliação da qualidade proposto por Donabedian 6 .
O processo de validação foi constituído por três fases: face a face, com a colaboração de dez pesquisadores da área; validação do conteúdo, por contraste, comparando-se usuários da rede privada e da pública; validação do constructo, divergente e convergente: a divergente baseouse na sensação de dor referida pelo usuário nos últimos seis meses, como medida de referência de saúde bucal pelo paciente, e a convergente tomou como base o grau de satisfação com a saúde bucal. O instrumento foi apurado mediante os métodos aditivo e dicotomizado, apresentando boa consistência interna. A apuração pelo método aditivo demonstrou melhores qualidades psicométricas, e a análise fatorial originou a versão que foi utilizada para este estudo, formada por 11 perguntas ou questões $(Q)$, abrangendo sete dimensões da qualidade: (1) relações humanas, referentes ao tratamento dispensado pelo dentista (Q1) e por outros profissionais (auxiliar do dentista-Q2); (2) eficácia, relacionada ao incômodo após o tratamento (Q3); (3) acessibilidade, apurada pela obtenção de uma vaga (Q4) e pelo tempo de espera no dia do atendimento (Q5); (4) qualidade técnico-científica, em relação à atualização tecnológica dos equipamentos odontológicos (Q6); (5) ambiente físico, ou seja, limpeza da recepção (Q7) e de banheiros (Q8); (6) aceitabilidade, referente à explicação ao usuário sobre o tratamento mais adequado (Q9); (7) resolutividade, relativa à satisfação com a aparência (Q10) e capacidade de mastigação dos dentes tratados (Q11). O instrumento foi construído com base na escala de Likert, com manifestações de respostas variando de excelente a péssimo, sendo tratadas como variáveis ordinais, cujos intervalos foram assumidos como regulares 22 .

No presente estudo, utilizou-se uma semana para a entrevista em um dos centros em cada mês dos quatro meses de coleta de dados. Ou seja, uma semana do mês para cada centro, totalizando quatro semanas, de meses diferentes, para cada centro. Para cada semana foram sorteados aleatoriamente 17 pacientes dentre aqueles agendados para o atendimento durante a semana utilizada. Foram excluídos os casos de pacientes menores de 18 anos, com necessidades especiais cognitivas que dificultassem a comunicação, ou ainda aqueles que estavam sendo assistidos no centro pela primeira vez. Dos pacientes previamente selecionados pela lista de agendamento, os que faltassem à consulta eram prontamente substituídos pelos próximos nomes da lista de agendamento. A coleta de dados foi realizada em diferentes horas do dia, contemplando diversas especialidades e profissionais, visando a oferecer uma amostra representativa do serviço. 
Os dados foram analisados por intermédio da freqüência e da utilização dos testes estatísticos qui-quadrado de Pearson ou teste exato de Fisher. O nível de significância utilizado na decisão dos testes estatísticos foi de $5 \%$. Os dados foram digitados na planilha Excel (Microsoft Corp., Estados Unidos) e exportados para o programa estatístico SPSS na versão 13.0 (SPSS Inc., Chicago, Estados Unidos).

A pesquisa foi submetida ao Comitê de Ética da Universidade Federal de Pernambuco, tendo sido aprovada sob o registro $\mathrm{n}^{\circ}$. 151.537 no Sistema Nacional de Informações sobre Ética em Pesquisa envolvendo Seres Humanos (SISNEP), em consonância com a Resolução $n^{\circ}$. 196/96 do Conselho Nacional de Saúde.

\section{Resultados}

\section{Características sócio-demográficas dos entrevistados}

Como mostra a Tabela 1, a idade dos usuários variou entre 18 e 74 anos, sendo os maiores percentuais correspondentes às idades entre $31 \mathrm{e}$ 50 anos $(40,8 \%)$ e entre 18 e 30 anos $(40,4 \%)$. A maioria entrevistada foi do sexo feminino (73,9\%), referindo a cor da pele parda $(51,8 \%)$, vivendo só (60,3\%) e com o nível de escolaridade até o Ensino Fundamental (54\%). No que se refere a trabalho/renda, evidenciou-se que a maior parte $(46 \%)$ possuía um trabalho esporádico, com renda variável. Também pode ser visto na Tabela 1 que a variável que apresentou maior diferença entre os Centros de Especialidades Odontológicas foi trabalho/renda, mas não de forma significativa no nível de $5 \%(p=0,06)$. A prevalência de usuários sem trabalho/renda no CEO D foi 16,2\%; nos CEO A e B, foi de 7,4\%.

\section{Avaliação das dimensões da qualidade pelo grupo total}

Os resultados das dimensões da qualidade no grupo total estão apresentados na Tabela 2. Eles mostram avaliações positivas pela maior parte dos usuários para as seguintes dimensões: relações humanas/cirurgião-dentista e auxiliar; eficácia/incômodo após o tratamento; qualidade técnico-científica/tecnologia do equipamento; aceitabilidade/explicação do tratamento pelo profissional.

A maioria dos entrevistados considerou excelente a atenção recebida pelo cirurgião-dentista (64\%) e auxiliar de saúde bucal (51,5\%); 84,9\% não sentiram nenhum incômodo após a realização do tratamento; $67,8 \%$ classificaram como modernos os equipamentos utilizados durante o atendimento; e $72,4 \%$ informaram sempre receber explicação sobre o tratamento.

No entanto, em relação à dimensão acessibilidade, a maioria das respostas dos usuários não corresponde a avaliações positivas. Na verdade, para a questão sobre obtenção de uma vaga, apesar de 29,8\% considerarem fácil obter uma vaga, $24,6 \%$ responderam ser difícil e 27,9\%, muito difícil, o que representou, após o somatório das duas últimas, 52,5\% de avaliações negativas. Em relação ao tempo de espera entre a chegada do usuário e o seu atendimento no dia da consulta, o maior percentual (48\%) correspondeu às opções pela resposta nem longo, nem curto.

Com relação à dimensão ambiente físico, a limpeza da recepção teve avaliação positiva pela maioria dos entrevistados, sendo classificada como boa por $57 \%$ da amostra; entretanto, a limpeza dos banheiros foi avaliada negativamente por boa parte dos entrevistados, sendo classificada como péssima por $34,8 \%$ dos respondentes, apesar de apenas 141 usuários terem se posicionado.

Por fim, quando se avaliou a dimensão resolutividade, os maiores percentuais foram observados para as respostas satisfeitos com a aparência $(40,3 \%)$ e com a capacidade de mastigação $(39,9 \%)$ dos dentes tratados no serviço. Por sua vez, o somatório do percentual de respostas totalmente satisfeitos e muito satisfeitos com a aparência correspondeu a 45,2\% e, em relação à capacidade de mastigação, o somatório foi de $37,7 \%$ da amostra. Isso demonstrou que boa parte dos usuários fez avaliações positivas também para essa dimensão da qualidade neste estudo.

\section{Avaliação das dimensões da qualidade por unidade de serviço/Centros de Especialidades Odontológicas}

Os resultados da Tabela 3 demonstram que existiu diferença significante entre as dimensões da qualidade abaixo relacionadas, quando foram avaliadas isoladamente, por unidade de serviço: relações humanas/cirurgião-dentista $(\mathrm{p}=0,001)$ e relações humanas/auxiliar de saúde bucal ( $p=0,007)$; eficácia/incômodo após o tratamento $(\mathrm{p}=0,014)$; acessibilidade/ tempo de espera pelo profissional no dia da consulta ( $\mathrm{p}=0,018)$; ambiente físico/limpeza da recepção ( $p=0,004)$; aceitabilidade/explicação do tratamento pelo profissional ( $\mathrm{p}=$ $0,029)$; efetividade/aparência dos dentes ( $\mathrm{p}=$ $0,029)$ e efetividade/capacidade de mastigação $(\mathrm{p}<0,001)$.

Nos quatro centros, a maioria dos usuários considerou a atenção recebida pelo cirurgião- 
Caracterização da amostra de 272 usuários atendidos nos centros de especialidades odontológicas do Município do Recife, Pernambuco, Brasil, 2008

\begin{tabular}{|c|c|c|c|c|c|c|c|c|c|c|c|}
\hline \multirow[t]{2}{*}{ Variável independente } & \multicolumn{2}{|c|}{ CEO A } & \multicolumn{2}{|c|}{ CEO B } & \multicolumn{2}{|c|}{ CEO C } & \multicolumn{2}{|c|}{ CEO D } & \multirow[t]{2}{*}{ Valor de $p$ * } & \multicolumn{2}{|c|}{ Grupo total } \\
\hline & $\mathrm{n}$ & $\%$ & $\mathrm{n}$ & $\%$ & $\mathbf{n}$ & $\%$ & $\mathrm{n}$ & $\%$ & & $\mathrm{n}$ & $\%$ \\
\hline \multicolumn{12}{|l|}{ Faixa etária (anos) } \\
\hline $18-30$ & 31 & 45,6 & 25 & 36,8 & 28 & 41,2 & 26 & 38,2 & 0,70 & 110 & 40,4 \\
\hline $31-50$ & 22 & 32,4 & 30 & 44,1 & 27 & 39,7 & 32 & 47,1 & & 111 & 40,8 \\
\hline $51-74$ & 15 & 22,0 & 13 & 19,1 & 13 & 19,1 & 10 & 14,7 & & 51 & 18,8 \\
\hline \multicolumn{12}{|l|}{ Sexo } \\
\hline Masculino & 17 & 25,0 & 17 & 25,0 & 17 & 25,0 & 20 & 29,4 & 0,91 & 71 & 26,1 \\
\hline Feminino & 51 & 75,0 & 51 & 75,0 & 51 & 75,0 & 48 & 70,6 & & 201 & 73,9 \\
\hline \multicolumn{12}{|l|}{ Raça } \\
\hline Branca & 26 & 38,2 & 25 & 36,8 & 21 & 30,9 & 25 & 36,8 & 0,28 & 97 & 35,7 \\
\hline Preta & 5 & 7,4 & 5 & 7,4 & 7 & 10,3 & 14 & 20,6 & & 31 & 11,4 \\
\hline Parda & 36 & 53,0 & 37 & 54,4 & 39 & 57,4 & 29 & 42,6 & & 141 & 51,8 \\
\hline Amarela & 1 & 1,4 & 0 & 0,0 & 1 & 1,4 & 0 & 0,0 & & 2 & 0,7 \\
\hline Indígena & 0 & 0,0 & 1 & 1,4 & 0 & 0,0 & 0 & 0,0 & & 1 & 0,4 \\
\hline \multicolumn{12}{|l|}{ Estado civil } \\
\hline Vive só ** & 42 & 61,8 & 39 & 57,4 & 38 & 55,9 & 45 & 66,2 & 0,54 & 164 & 60,3 \\
\hline Vive com alguém *** & 26 & 38,2 & 29 & 42,6 & 30 & 44,1 & 22 & 32,4 & & 107 & 39,3 \\
\hline Não informou & 0 & 0,0 & 0 & 0,0 & 0 & 0,0 & 0 & 1,4 & & 1 & 0,4 \\
\hline \multicolumn{12}{|l|}{ Escolaridade } \\
\hline Até o Ensino Fundamental & 39 & 58,0 & 31 & 45,6 & 44 & 64,7 & 33 & 49,1 & 0,11 & 147 & 54,0 \\
\hline Ensino Médio/Superior & 28 & 41,7 & 37 & 54,4 & 24 & 35,3 & 34 & 50,6 & & 123 & 45,2 \\
\hline Não informou & 1 & 0,3 & 0 & 0,0 & 0 & 0,0 & 1 & 0,3 & & 2 & 0,7 \\
\hline \multicolumn{12}{|l|}{ Trabalho/Renda } \\
\hline Sem trabalho/Sem renda & 5 & 7,4 & 5 & 7,4 & 7 & 10,3 & 11 & 16,2 & 0,06 & 28 & 10,3 \\
\hline Com trabalho/Renda fixa & 23 & 33,8 & 31 & 45,6 & 29 & 42,6 & 36 & 52,9 & & 119 & 43,8 \\
\hline Trabalho esporádico/Renda variável & 40 & 58,8 & 32 & 47,0 & 32 & 47,1 & 21 & 30,9 & & 125 & 46,0 \\
\hline Total & 68 & 100,0 & 68 & 100,0 & 68 & 100,0 & 68 & 100,0 & & 272 & 100,0 \\
\hline
\end{tabular}

* Comparação da distribuição das variáveis entre os centros de especialidades odontológicas;

** Incluindo solteiro, separado, desquitado, viúvo;

*** Incluindo casado e união estável.

dentista entre excelente e boa, destacando-se o CEO B por ter sido o único a obter $100 \%$ de satisfação do usuário nessa dimensão. Quando a avaliação foi da relação auxiliar de saúde bucal/ paciente, nenhum dos centros alcançou o grau máximo de satisfação, e o CEO A foi o responsável pelo maior índice $(7,8 \%)$ de pacientes que classificou como ruim ou péssima essa relação.

Em relação à dimensão eficácia, apesar de a resposta mais positiva (não ter sentido nenhum incômodo após a realização do tratamento) também ter correspondido aos maiores percentuais em todos os centros, no CEO D o percentual foi menor $(77,9 \%)$, pois $19,1 \%$ dos entrevistados ainda referiram um pouco de desconforto após o tratamento nesse centro. No entanto, foi novamente do CEO A o maior percentual $(10,3 \%)$ dos que estavam insatisfeitos; nesse caso, porque sentiram muito desconforto.

Na questão sobre tempo de espera pelo profissional no dia da consulta (dimensão acessibilidade), os resultados não foram totalmente positivos, nem totalmente negativos para os centros avaliados. Os usuários do CEO C foram os que se declararam mais satisfeitos, visto que o maior percentual $(33,8 \%)$ achou o tempo de espera curto ou muito curto. Contrariamente, no CEO B foram destacados os usuários mais insatisfeitos, pois, para $38,2 \%$ deles, o tempo de espera foi longo ou muito longo.

Na dimensão ambiente físico, a limpeza da sala de espera foi satisfatória para a maioria dos usuários dos CEO A, B, C e D. Particularmente no CEO C, nenhum paciente ficou insatisfeito com 
Tabela 2

Avaliação da satisfação dos usuários assistidos nos Centros de Especialidades Odontológicas do Município do Recife, Pernambuco, Brasil, 2008, segundo as dimensões da qualidade propostas por Donabedian 6 e adaptadas por Fernandes 22.

\begin{tabular}{|c|c|c|c|c|c|c|}
\hline \multirow{2}{*}{$\begin{array}{l}\text { Dimensão de qualidade } \\
\text { Dimensão } 1 \text { - relações humanas }\end{array}$} & \multicolumn{6}{|c|}{ Satisfação do usuário (escore) } \\
\hline & Excelente (5) & Boa (4) & Regular (3) & Ruim (2) & Péssima (1) & Total \\
\hline & $n(\%)$ & n (\%) & n (\%) & n (\%) & n (\%) & n (\%) \\
\hline Cirurgião-dentista & $174(64,0)$ & $84(30,9)$ & $11(4,0)$ & $2(0,7)$ & $1(0,4)$ & $272(100,0)$ \\
\hline Auxiliar de saúde bucal & $137(51,5)$ & $106(39,8)$ & $17(6,4)$ & $4(1,5)$ & $2(0,7)$ & $266 *(100,0)$ \\
\hline \multirow{3}{*}{ Dimensão 2 - eficácia } & Não senti & Pouco & Senti & Muito & Total & Total \\
\hline & nada (5) & desconforto (4) & desconforto (3) & desconforto (2) & desconforto (1) & \\
\hline & $n(\%)$ & n (\%) & n (\%) & n (\%) & n (\%) & n (\%) \\
\hline Incômodo & $231(84,9)$ & $21(7,7)$ & $9(3,3)$ & $7(2,6)$ & $4(1,5)$ & $272(100,0)$ \\
\hline \multirow[t]{3}{*}{ Dimensão 3 - acessibilidade } & Muito fácil (5) & Fácil (4) & Nem fácil nem & Difícil (2) & Muito difícil (1) & Total \\
\hline & & & difícil (3) & & & \\
\hline & n (\%) & n (\%) & n (\%) & n (\%) & n (\%) & $n(\%)$ \\
\hline \multirow[t]{4}{*}{ Obtenção de vaga } & $5(1,8)$ & $81(29,8)$ & $43(15,8)$ & $67(24,6)$ & $76(27,9)$ & $272(100,0)$ \\
\hline & Muito curto (5) & Curto (4) & Nem longo & Longo (2) & Muito & Total \\
\hline & & & nem curto (3) & & longo (1) & \\
\hline & n (\%) & n (\%) & n (\%) & n (\%) & n (\%) & n (\%) \\
\hline Tempo de espera & $7(2,6)$ & $60(22,1)$ & $130(48,0)$ & $55(20,3)$ & $19(7,0)$ & $271 * \star(100,0)$ \\
\hline \multirow{3}{*}{$\begin{array}{l}\text { Dimensão } 4 \text { - qualidade técnico- } \\
\text { científica }\end{array}$} & Super & Moderno (4) & Mais ou & Desatualizado & Ultrapassado (1) & Total \\
\hline & moderno (5) & & menos (3) & (2) & & \\
\hline & n (\%) & n (\%) & n (\%) & n (\%) & n (\%) & $n(\%)$ \\
\hline Equipamento & $18(6,7)$ & $181(67,8)$ & $61(22,8)$ & $6(2,2)$ & $1(0,4)$ & $267^{\star \star \star}(100,0)$ \\
\hline Dimensão 5 - ambiente físico/ & Excelente (5) & Boa (4) & Regular (3) & Ruim (2) & Péssima (1) & Total \\
\hline limpeza & n (\%) & n (\%) & n (\%) & n (\%) & n (\%) & n (\%) \\
\hline Recepção & $14(5,1)$ & $155(57,0)$ & $87(32,0)$ & $7(2,6)$ & $9(3,3)$ & $272(100,0)$ \\
\hline Banheiro & $1(0,7)$ & $30(21,3)$ & $47(33,3)$ & $14(9,9)$ & $49(34,8)$ & $141 \#(100,0)$ \\
\hline \multirow[t]{3}{*}{ Dimensão 6 - aceitabilidade } & Sempre me & Na maiorida & Não me & Raras vezes me & Nunca me & Total \\
\hline & explica tudo (5) & $\begin{array}{c}\text { das vezes me } \\
\text { explica (4) }\end{array}$ & lembro (3) & explica (2) & explica (1) & \\
\hline & n (\%) & n (\%) & n (\%) & n (\%) & n (\%) & n (\%) \\
\hline Explicação do tratamento & $197(72,4)$ & $16(5,9)$ & $1(0,4)$ & $12(4,4)$ & $46(16,9)$ & $272(100,0)$ \\
\hline \multirow[t]{3}{*}{ Dimensão 7 - resolutividade } & Totalmente & Muito & Satisfeito (3) & Pouco & Insatisfeito (1) & Total \\
\hline & satisfeito (5) & satisfeito (4) & & satisfeito (2) & & \\
\hline & n (\%) & n (\%) & $n(\%)$ & $n(\%)$ & n (\%) & n (\%) \\
\hline Aparência dos dentes & $45(16,8)$ & $76(28,4)$ & $108(40,3)$ & $23(8,6)$ & $16(6,0)$ & $268 \#(100,0)$ \\
\hline Capacidade de mastigação & $52(19,2)$ & $50(18,5)$ & $108(39,9)$ & $34(12,5)$ & $27(10,0)$ & $271 \star \star(100,0)$ \\
\hline
\end{tabular}

* Para 6 usuários não se dispõe desta informação;

** Para 1 usuário não se dispõe desta informação;

*** Para 5 usuários não se dispõe desta informação;

\# Para 131 usuários não se dispõe desta informação;

\#\# Para 4 usuários não se dispõe desta informação.

essa dimensão, uma vez que ninguém classificou a limpeza como ruim ou péssima; já o CEO A foi o que apresentou maior percentual de usuários insatisfeitos com a limpeza desse ambiente, com $11,8 \%$ classificando-a como ruim ou péssima.

De acordo com os resultados, a maioria dos usuários de cada centro também estava satisfeita com a dimensão aceitabilidade. O CEO B apre- sentou, simultaneamente, o maior índice $(88,2 \%)$ de pacientes aos quais o profissional sempre ou na maioria das vezes explicava tudo sobre o tratamento e o menor (10,3\%), referente àqueles que raras vezes ou nunca recebiam tais informações. Inversamente ocorreu com o CEO C, com menor percentual de usuários satisfeitos. 
Tabela 3

Avaliação individualizada da satisfação dos usuários, por Centro de Especialidade Odontológica do Município do Recife, Pernambuco, Brasil, 2008, segundo as dimensões da qualidade propostas por Donabedian 6 e adaptadas por Fernandes 22.

\begin{tabular}{|c|c|c|c|c|c|c|c|c|c|}
\hline \multirow[t]{2}{*}{ Dimensão da qualidade } & \multicolumn{2}{|c|}{ CEO A } & \multicolumn{2}{|c|}{ CEO B } & \multicolumn{2}{|c|}{ CEO C } & \multicolumn{2}{|c|}{ CEO D } & \multirow[t]{2}{*}{ Valor de $p$} \\
\hline & $\mathbf{n}$ & $\%$ & $\mathrm{n}$ & $\%$ & n & $\%$ & $\mathrm{n}$ & $\%$ & \\
\hline \multicolumn{10}{|l|}{ Dimensão 1 - relações humanas } \\
\hline \multicolumn{10}{|l|}{ Cirurgião-Dentista } \\
\hline Excelente/Boa & 62 & 91,2 & 68 & 100,0 & 67 & 98,5 & 61 & 89,7 & $0,001 *, \star \star$ \\
\hline Regular & 4 & 5,9 & - & - & - & - & 7 & 10,3 & \\
\hline Ruim/Péssima & 2 & 2,9 & - & - & 1 & 1,5 & - & - & \\
\hline Total & 68 & 100,0 & 68 & 100,0 & 68 & 100,0 & 68 & 100,0 & \\
\hline \multicolumn{10}{|l|}{ Auxiliar de saúde bucal } \\
\hline Excelente/Boa & 53 & 82,8 & 60 & 88,2 & 67 & 98,5 & 63 & 95,5 & $0,007 *, \star \star$ \\
\hline Regular & 6 & 9,4 & 7 & 10,3 & 1 & 1,5 & 3 & 4,5 & \\
\hline Ruim/Péssima & 5 & 7,8 & 1 & 1,5 & - & - & - & - & \\
\hline Total & 64 & 100,0 & 68 & 100,0 & 68 & 100,0 & 66 & 100,0 & \\
\hline \multicolumn{10}{|l|}{ Dimensão 2 - eficácia } \\
\hline \multicolumn{10}{|l|}{ Incômodo } \\
\hline Não sentiu nada & 57 & 83,8 & 61 & 89,7 & 60 & 88,2 & 53 & 77,9 & $0,014 *, \star \star$ \\
\hline Pouco desconforto/Desconforto & 4 & 5,9 & 5 & 7,4 & 8 & 11,8 & 13 & 19,1 & \\
\hline Muito desconforto/Desconforto total & 7 & 10,3 & 2 & 2,9 & - & - & 2 & 2,9 & \\
\hline Total & 68 & 100,0 & 68 & 100,0 & 68 & 100,0 & 68 & 100,0 & \\
\hline \multicolumn{10}{|l|}{ Dimensão 3 - acessibilidade } \\
\hline \multicolumn{10}{|l|}{ Obtenção de vaga } \\
\hline Fácil/Muito fácil & 30 & 44,1 & 20 & 29,4 & 18 & 265 & 18 & 26,5 & $0,273 * \star \star *$ \\
\hline Nem fácil, nem difícil & 11 & 16,2 & 10 & 14,7 & 11 & 16,2 & 11 & 16,2 & \\
\hline Difícil/Muito difícil & 27 & 39,7 & 38 & 55,9 & 39 & 57,4 & 39 & 57,4 & \\
\hline Total & 68 & 100,0 & 68 & 100,0 & 68 & 100,0 & 68 & 100,0 & \\
\hline \multicolumn{10}{|l|}{ Tempo de espera } \\
\hline Curto/Muito curto & 17 & 25,0 & 10 & 14,7 & 23 & 33,8 & 17 & 25,4 & 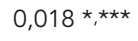 \\
\hline Nem longo, nem difícil & 28 & 41,2 & 32 & 47,1 & 31 & 45,6 & 39 & 58,2 & \\
\hline Longo/Muito longo & 23 & 33,8 & 26 & 38,2 & 14 & 20,6 & 11 & 16,4 & \\
\hline Total & 68 & 100,0 & 68 & 100,0 & 68 & 100,0 & 67 & 100,0 & \\
\hline \multicolumn{10}{|l|}{ Dimensão 4 - qualidade técnico-científica } \\
\hline \multicolumn{10}{|l|}{ Equipamento } \\
\hline Moderno/Super moderno & 51 & 81,0 & 45 & 66,2 & 51 & 75,0 & 52 & 76,5 & $0,385 * \star$ \\
\hline Mais ou menos & 12 & 19,0 & 21 & 30,9 & 14 & 20,6 & 14 & 20,6 & \\
\hline Ultrapassado/Desatualizado & - & - & 2 & 2,9 & 3 & 4,4 & 2 & 2,9 & \\
\hline Total & 63 & 100,0 & 68 & 100,0 & 68 & 100,0 & 68 & 100,0 & \\
\hline \multicolumn{10}{|l|}{ Dimensão 5 - ambiente físico/limpeza } \\
\hline \multicolumn{10}{|l|}{ Recepção } \\
\hline Excelente/Boa & 32 & 47,1 & 42 & 61,8 & 51 & 75,0 & 43 & 63,2 &  \\
\hline Regular & 28 & 41,2 & 24 & 35,3 & 17 & 25,0 & 19 & 27,9 & \\
\hline Ruim/Péssima & 8 & 11,8 & 2 & 2,9 & - & - & 6 & 8,8 & \\
\hline Total & 68 & 100,0 & 68 & 100,0 & 68 & 100,0 & 68 & 100,0 & \\
\hline \multicolumn{10}{|l|}{ Banheiro } \\
\hline Excelente/Boa & 8 & 17,8 & 8 & 16,3 & 12 & 38,7 & 3 & 18,8 & 0,291 *** \\
\hline Regular & 15 & 33,3 & 17 & 34,7 & 10 & 32,3 & 5 & 31,3 & \\
\hline Ruim/Péssima & 22 & 48,9 & 24 & 49,0 & 9 & 29,0 & 8 & 50,0 & \\
\hline Total & 45 & 100,0 & 49 & 100,0 & 31 & 100,0 & 16 & 100,0 & \\
\hline
\end{tabular}

(continua) 
Tabela 3 (continuação)

\begin{tabular}{|c|c|c|c|c|c|c|c|c|c|}
\hline \multirow[t]{2}{*}{ Dimensão da qualidade } & \multicolumn{2}{|c|}{ CEO A } & \multicolumn{2}{|c|}{ CEO B } & \multicolumn{2}{|c|}{ CEO C } & \multicolumn{2}{|c|}{ CEO D } & \multirow[t]{2}{*}{ Valor de $\mathrm{p}$} \\
\hline & $\mathbf{n}$ & $\%$ & $\mathbf{n}$ & $\%$ & $\mathbf{n}$ & $\%$ & $\mathbf{n}$ & $\%$ & \\
\hline \multicolumn{10}{|l|}{ Dimensão 6 - aceitabilidade } \\
\hline \multicolumn{10}{|l|}{ Explicação do tratamento } \\
\hline Sempre/Na maioria das vezes explica & 54 & 79,5 & 60 & 88,2 & 47 & 69,1 & 52 & 76,5 & $0,029 *, \star \star *$ \\
\hline Não me lembro & - & - & 1 & 1,5 & - & - & - & - & \\
\hline Raras vezes/Nunca explica & 14 & 20,5 & 7 & 10,3 & 21 & 30,9 & 16 & 23,5 & \\
\hline Total & 68 & 100,0 & 68 & 100,0 & 68 & 100,0 & 68 & 100,0 & \\
\hline \multicolumn{10}{|l|}{ Dimensão 7 - resolutividade } \\
\hline \multicolumn{10}{|l|}{ Aparência dos dentes } \\
\hline Muito/Totalmente satisfeito & 27 & 41,5 & 35 & 51,5 & 28 & 41,8 & 31 & 45,6 & $0,029 *, \star \star$ \\
\hline Satisfeito/Um pouco satisfeito & 34 & 52,3 & 30 & 44,1 & 39 & 58,2 & 28 & 41,2 & \\
\hline Insatisfeito & 4 & 6,2 & 3 & 4,4 & - & - & 9 & 13,2 & \\
\hline Total & 65 & 100,0 & 68 & 100,0 & 67 & 100,0 & 68 & 100,0 & \\
\hline \multicolumn{10}{|l|}{ Capacidade de mastigação } \\
\hline Muito/Totalmente satisfeito & 25 & 37,3 & 23 & 33,8 & 15 & 22,1 & 39 & 57,4 & $<0,001 *$, \\
\hline Satisfeito/Um pouco satisfeito & 34 & 50,7 & 42 & 61,8 & 49 & 72,1 & 17 & 25,0 & \\
\hline Insatisfeito & 8 & 11,9 & 3 & 4,4 & 4 & 5,9 & 12 & 17,6 & \\
\hline Total & 67 & 100,0 & 68 & 100,0 & 68 & 100,0 & 68 & 100,0 & \\
\hline
\end{tabular}

* Diferença significante de 5\%;

** Através do teste exato de Fisher;

*** Através do teste de qui-quadrado de Pearson.

Na dimensão resolutividade/aparência dos dentes tratados, há que se destacar que, apesar de somente no CEO B a maioria dos usuários ter informado estar muito ou totalmente satisfeita (51,5\%), foi exclusivamente no CEO C onde nenhum usuário declarou estar insatisfeito. Já em relação à resolutividade/ capacidade de mastigação, o CEO D apresentou uma situação interessante: foi, ao mesmo tempo, o único centro onde a maioria $(57,4 \%)$ estava muito ou totalmente satisfeita com a capacidade de mastigação e onde se observou o maior percentual de usuários insatisfeitos nessa dimensão (17,6\%). Dentre os centros, foi o CEO B que apresentou o menor percentual de insatisfeitos com a capacidade de mastigação do dente tratado $(4,4 \%)$.

\section{Discussão}

No presente estudo, foram contempladas sete dimensões da qualidade para a avaliação de serviços sob a ótica dos usuários: relações humanas, eficácia, acessibilidade, qualidade técnico-científica, aceitabilidade, ambiente físico e resolutividade.

Em relação à satisfação do usuário, foi detectada uma boa avaliação dos Centros de Especialidades Odontológicas do Município do Recife, uma vez que a satisfação do paciente pode ser definida como as avaliações positivas individuais de distintas dimensões do cuidado à saúde 24 Entretanto, algumas diferenças entre os centros foram observadas, e a acessibilidade surgiu como uma dificuldade enfrentada pelos usuários de forma geral.

Quanto à metodologia utilizada, concorda-se com Fernandes 22 que o QASSaB é um instrumento prático e válido para quantificar a qualidade dos serviços de saúde bucal. No entanto, apesar de alguns autores questionarem a consistência dos comentários dos pacientes 16, considera-se importante a criação de um espaço para comentários e sugestões destes, pois tais registros podem apresentar dados importantes e complementares à avaliação quantitativa 10,17 .

A avaliação positiva de serviços sob a ótica dos usuários também foi resultado encontrado em outras pesquisas 14,22. Além disso, o receio de sanção no atendimento ${ }^{17}$, o gratitude bias, ou seja, o sentimento de gratidão do usuário pelo serviço gratuito 16,25 , a impossibilidade de acesso a outro serviço, ou a proximidade do posto à sua residência 16 constituem fatores que explicariam tal avaliação por pacientes de países cujos serviços públicos de atenção à saúde são predominantemente utilizados por usuários de baixa renda. 
Especificamente em relação aos Centros de Especialidades Odontológicas do Município do Recife, os resultados positivos da avaliação pelo usuário coincidiram com os resultados positivos de uma avaliação normativa. Da mesma forma que os usuários se declararam satisfeitos com os serviços oferecidos, esses centros, tipo II, localizados em cidades habilitadas na gestão plena do sistema do Estado de Pernambuco, funcionando em serviços pré-existentes, apresentaram um bom desempenho na avaliação técnica 21 .

Na presente pesquisa, assim como em outros estudos 14,16,17,22, a dimensão relações humanas foi motivo de satisfação dos usuários. A atenção do profissional e o tempo de duração da consulta podem ter originado uma associação positiva, sendo influenciada ou influenciando a comunicação estabelecida entre profissional e paciente. Essa correlação positiva entre os fatores foi referida numa pesquisa de avaliação da relação médico-paciente, sob a ótica do paciente, na rede pública de saúde do Brasil 26. Em adição, expressam a opinião de alguns autores 16 quando consideram que a relação profissional-paciente tende a ser insatisfatória se houver limitação do tempo da consulta.

Os resultados deste estudo, contudo, revelaram um profissional com um perfil diferente, que dialoga com os pacientes, explicando-lhes o tratamento a ser realizado (aceitabilidade) e que desenvolve uma relação empática e participativa com eles (relações humanas). Sendo assim, um maior tempo para consulta devido à cota de atendimento por profissional nos Centros de Especialidades Odontológicas pode possibilitar um tempo maior para a escuta do paciente. Isso facilitaria o processo de humanização do atendimento, extremamente relevante para a satisfação do usuário 9,27. Ademais, o profissional de saúde tem que se esforçar para mudar sua relação com o saber e com o conjunto de atores sociais envolvidos, especialmente o usuário do serviço, uma vez que o atendimento baseado no estilo médico-centrado 16 não satisfaz mais as expectativas do paciente. Este, atualmente, espera do profissional de saúde uma situação de acolhimento 28 capaz não só de aliviar e amenizar seu sofrimento, como também de responder resolutivamente ao seu problema de saúde 29 .

Por outro lado, o número de profissionais por especialidades nos centros pode ser insuficiente, pois a obtenção de uma vaga foi considerada difícil pela maioria dos usuários, sugerindo que, no âmbito do Sistema Único de Saúde (SUS), a expansão da rede assistencial de atenção odontológica secundária não acompanhou o crescimento da oferta de serviços da atenção básica, podendo gerar insatisfação. Em concordância, dados do
Sistema de Informações Ambulatoriais do SUS (SIA/SUS) informam que os serviços odontológicos especializados correspondem a não mais do que $3,5 \%$ do total de procedimentos clínicos odontológicos realizados 30 .

Longas filas de espera, necessidade de chegar muito cedo para obtenção de uma ficha e favorecimento de vagas no serviço de saúde 19, assim como cobertura insuficiente, demanda reprimida e o fato de ser usuário exclusivo da rede pública ${ }^{13}$, podem estar intimamente relacionados à percepção do usuário sobre a dificuldade para obtenção de uma vaga para o atendimento. Na saúde bucal, a dificuldade de acesso aos serviços odontológicos no Município do Recife foi observada quando da avaliação de serviços de urgência 20 , tendo sido também destacada como um dos principais problemas de saúde bucal no Brasil 31.

Quando se trata de comparar a qualidade dos serviços prestados em cada Centro de Especialidade Odontológica por meio da satisfação do usuário, há que se destacar positivamente os CEO B e C, onde um ou outro obteve os maiores percentuais de mais alto nível de satisfação em quase todas as dimensões, dentre aquelas que apresentaram diferença significativa entre os centros, excetuando-se o componente capacidade de mastigação da dimensão resolutividade. Mesmo nesse componente, o CEO B obteve o menor percentual de indivíduos com menor nível de satisfação. Por outro lado, o CEO A carece de maior atenção dos gestores, tendo em vista que foi nele que se alcançaram os maiores percentuais, ou os segundos maiores, de mais baixo nível de satisfação em boa parte das dimensões avaliadas.

As maiores diferenças encontradas entre os centros $(\mathrm{p}<0,01)$ apresentaram-se na dimensão relações humanas, no componente recepção da dimensão ambiente físico e no componente capacidade de mastigação da dimensão resolutividade, indicando que intervenções devem ser feitas em termos tanto de recursos humanos, quanto de infra-estrutura.

A percepção individual do usuário sobre o seu papel no sistema de saúde 27 e as características sociais e demográficas desse usuário 13,22 podem influenciar na sua satisfação com serviços de saúde. Considerando-se que, no CEO D, a prevalência dos usuários sem trabalho ou renda foi maior que o dobro do encontrado nos CEO A e B, a condição de não ter trabalho pode ter afetado a avaliação do usuário em face das diversas dimensões da qualidade. Observe-se ainda que os usuários do CEO D fizeram as avaliações mais positivas quanto ao tempo de espera e na categoria ambiente físico/limpeza da recepção, 
com a menor proporção das respostas longo/ muito longo e ruim/péssimo. Por outro lado, os usuários do CEO C, que apresentaram a pior condição de escolaridade, relataram a melhor avaliação das relações humanas para auxiliar. Apesar de, no processo de validação do QAASaB 22, as características sócio-demográficas, idade e renda terem sido associadas à satisfação do usuário de diferentes tipos de serviço, privado e público, em um outro estudo em Recife não foi observada uma relação estatisticamente significante entre as variáveis independentes e o nível de satisfação do usuário de serviços públicos de urgência 20.

Obviamente, mesmo que não se tenha atingido valor de $\mathrm{p}<0,05$, não se pode negligenciar $\mathrm{o}$ fato de que as diferenças na avaliação da satisfação dos usuários entre os Centros de Especialidades Odontológicas (Tabela 3) pode ter ocorrido, ao menos em parte, pelos diferentes perfis sócioeconômicos de seus usuários. Além disso, outras variáveis sócio-demográficas não analisadas no presente estudo, incluindo a dos profissionais e de infra-estrutura e administração, podem ter tido influência na diferença encontrada entre os centros, mas, para a resposta adequada a essa questão, seria necessária a realização de outro estudo, com delineamento específico, no qual seria possível tratar-se com profundidade toda a complexidade que o tema exige, analisando, por exemplo, uma gama bem maior de variáveis.

\section{Considerações finais}

Os Centros de Especialidades Odontológicas do Município do Recife são serviços novos, em construção, apresentando particularidades quando avaliados isoladamente.

Os resultados desta pesquisa ressaltaram o caráter multidimensional da avaliação dos serviços de saúde, sugerindo que a qualidade percebida pelo paciente pode estar muito mais relacionada à maneira como ele é tratado do que com os aspectos técnicos da assistência propriamente ditos. Sendo assim, foi possível reconhecer a relevância deste estudo em dois aspectos: primeiro por haver discreto número de pesquisas sobre avaliação de serviços da atenção secundária de saúde bucal no Brasil; segundo, pelo fato de a satisfação do usuário constituir uma maneira democrática de avaliação, possibilitando a participação social nos serviços de saúde preconizada pela reforma sanitária brasileira.

Portanto, novas pesquisas enfocando a satisfação do usuário deveriam ser realizadas como forma de colaborar na detecção de pontos de melhoria dos serviços e também na implementação de ações em prol de uma assistência de qualidade, humana e com resolutividade. 


\section{Resumo}

O estudo objetivou avaliar a satisfação dos usuários assistidos nos Centros de Especialidades Odontológicas do Município do Recife, Pernambuco, Brasil. Realizouse entrevista pessoal padronizada com 272 pacientes utilizando o Questionário de Avaliação da Qualidade dos Serviços de Saúde Bucal (QASSaB). Os resultados indicaram uma avaliação positiva para a maioria das dimensões no geral. A acessibilidade foi um ponto crítico destacado. Houve diferença significante de 5\% na satisfação dos usuários, entre as unidades de serviço, para: relações humanas/cirurgião-dentista $(p=0,001)$; relações humanas/auxiliar ( $p=0,007)$; eficácia ( $p=$ $0,014)$; acessibilidade/tempo de espera ( $p=0,018)$; ambiente físico/limpeza da recepção ( $p=0,004)$; aceitabilidade ( $p=0,029)$; efetividadelaparência dos dentes ( $p=$ $0,029)$ e efetividade/capacidade de mastigação $(p<$ 0,001). Concluiu-se que a maioria dos usuários está satisfeita com a assistência prestada nos centros e que a satisfação difere em cada centro.

Satisfação do Paciente; Qualidade da Assistência à Saúde; Saúde Bucal

\section{Colaboradores}

A. C. S. Lima participou da coleta e análise dos dados; redação e revisão crítica do artigo. M. M. V. B. Vasconcelos colaborou na revisão crítica do artigo. E. D. Cabral contribuiu no delineamento da metodologia, análise dos dados e revisão crítica do artigo.

\section{Referências}

1. Ministério da Saúde. Portaria no ${ }^{\circ}$ 599. Define a im plantação de especialidades odontológicas (CEO) e de laboratórios regionais de próteses dentárias (LRPDs) e estabelece critérios, normas e requisitos para seu credenciamento. Diário Oficial da União 2006; 24 mar.

2. Ministério da Saúde. Portaria $n^{\circ}$. 600. Institui o financiamento dos centros de especialidades odontológicas. Diário Oficial da União 2006; 24 mar.

3. Malik AM. Qualidade em serviços de saúde nos setores público e privado. Cad FUNDAP 1996; 19:7-24.

4. Souto LR, Espírito Santo ACG, Mendonça M, Cavalcanti Z. Percepção da clientela acerca dos fatores críticos no atendimento ambulatorial prestado pelo Hospital das Clínicas da UFPE. Rev IMIP 1996; 10:103-10

5. Williams B. Patient satisfaction: a valid concept? Soc Sci Med 1994; 38:509-16.
6. Donabedian A. The seven pillars of quality. Arch Pathol Lab Med 1990; 114:1115-8.

7. Vuori H. A qualidade de saúde. Divulg Saúde Debate 1991; (3):17-25.

8. Carr-Hill RA. The measurement of patient satisfaction. J Public Health Med 1992; 14:236-49.

9. Kloetzel K, Bertoni AM, Irazoqui MC, Campos VPG, Santos RN. Controle de qualidade em atenção primária à saúde. I - a satisfação do usuário. Cad Saúde Pública 1998; 14: 263-8.

10. Bosi MLM, Uchimura KY. Avaliação da qualidade ou avaliação qualitativa do cuidado em saúde? Rev Saúde Pública 2007; 41:150-3.

11. Mezomo JC. Qualidade na relação hospital-paciente. Hosp Adm Saúde 1993; 17:17-21.

12. Blendon RJ, Kim M, Benson JM. The public versus the world health organization on health system performance. Health Aff 2001; 20:11-20. 
13. Gouveia GC, Souza WV, Luna CF, Souza-Júnior PRB, Szwarcwald CL. Health care users' satisfaction in Brazil, 2003. Cad Saúde Pública 2005; 21 Suppl 1:S109-18.

14. Bottan ER, Sperb RAL, Telles OS, Uriarte NM. Avaliação de serviços odontológicos: a visão dos pacientes. Rev ABENO 2006; 6:128-33.

15. Souza RK, Scholtz MR. Utilização e avaliação dos serviços oferecidos por uma unidade básica de saúde do município de Maringá-PR. Saúde Debate 1996; (52):35-41.

16. Traverso-Yépez M, Morais NA. Reivindicando a subjetividade dos usuários da Rede Básica de Saúde: para uma humanização do atendimento. Cad Saúde Pública 2004; 20:80-8.

17. Andrade KLC, Ferreira EF. Avaliação da inserção da odontologia no Programa de Saúde da Família de Pompéu/MG: a satisfação do usuário. Ciênc Saúde Coletiva 2006; 11:123-30.

18. Leão ATT, Dias K. Avaliação dos serviços de saúde prestados por faculdades de odontologia: a visão do usuário. Revista Brasileira de Odontologia e Saúde Coletiva 2001; 2:40-6.

19. Lima MADS. Acesso e acolhimento em unidades de saúde na visão dos usuários. Acta Paul Enferm 2007; 20:12-7.

20. Carnut L, Figueiredo N, Goes PSA. Avaliação do nível de satisfação dos usuários das urgências odontológicas da cidade do Recife. UFES Rev Odontol 2008; 10:10-5.

21. Figueiredo N, Goes PSA. Construção da atenção secundária em saúde bucal: um estudo sobre os Centros de Especialidades Odontológicas em Pernambuco, Brasil. Cad Saúde Pública 2009; 25:259-67.

22. Fernandes LMAG. Validação de um instrumento para avaliação da satisfação dos usuários, com os serviços públicos de saúde bucal [Tese de Doutorado]. Camaragibe: Faculdade de Odontologia, Universidade de Pernambuco; 2002.
23. Prefeitura do Recife. Plano municipal de saúde plurianual 2006/2009. http://www.recife.pe.gov. br/modelo.php?id=138\&Tipo=D (acessado em 12/ Fev/2009)

24. Linder-Pelz S. Toward a theory of patient satisfaction. Soc Sci Med 1982; 16:577-82.

25. Vaitsman J, Andrade GRB. Satisfação e responsividade: formas de medir a qualidade e a humanização da assistência à saúde. Ciênc Saúde Coletiva 2005; 10:599-612.

26. Pereira MGA, Azevêdo ES. A relação médico-paciente em Rio branco/AC sob a ótica dos pacientes. Rev Assoc Med Bras 2005; 51:153-7.

27. Akerman A, Nadanovsky P. Avaliação dos serviços de saúde: avaliar o quê? Cad Saúde Pública 1992; 8:361-5.

28. Santos AM, Assis MMA, Rodrigues AAAO, Nascimento MAA, Jorge MSB. Linhas de tensões no processo de acolhimento das equipes de saúde bucal do Programa Saúde da Família: o caso de Alagoinhas, Bahia, Brasil. Cad Saúde Pública 2007; 25: 75-85.

29. Merphy EE. Em busca da qualidade dos serviços de saúde: os serviços de porta aberta para a saúde e o modelo tecno-assistencial em defesa da vida. In: Cecílio LC, organizador. Inventando a mudança na saúde. São Paulo: Editora Hucitec; 1997. p. 117-60.

30. Costa JFR, Chagas L, Silvestre R. A Política Nacional de Saúde Bucal do Brasil: registro de uma conquista histórica. Brasília: Organização Pan-Americana da Saúde; 2006. (Série Técnica: Desenvolvimento de Sistemas e Serviços de Saúde, 11).

31. Pinheiro RS, Torres TZG. Uso de serviços odontológicos entre os Estados do Brasil. Ciênc Saúde Coletiva 2006; 11:999-1010.

Recebido em 28/Abr/2009

Versão final reapresentada em 04/Mar/2010

Aprovado em 11/Mar/2010 